\title{
M.A. Proytcheva (ed): Diagnostic pediatric hematopathology
}

\author{
Cambridge University Press 2011, ISBN 978-0-521-88160-9
}

\author{
Konnie Hebeda
}

Published online: 8 September 2011

(C) Springer-Verlag 2011

Some books fill a gap on the bookshelf. This comprehensive textbook belongs to those. It provides an overview of the state-of-the-art of both non-neoplastic and neoplastic hematopathology in children, and incorporates recent developments in classifications, based on the 2008 WHO classification, and in understanding of the molecular biology of hematological disease. The abundant highquality photographs and schematic drawings encourage the use as a tool for daily hematopathological practice. For pathologists with less experience with pediatric or hematological cases, this book is an essential guide that not only provides an overview of hematology, but also focuses on the specific features of the normal and abnormal pediatric hematopoietic and lymphatic system. General introductions cover age-related changes and extensive reference tables for hematological and lymphoid values, and provide practical explanations of diagnostic techniques as cytogenetics, FISH, and expression profiling.

The chapters of the book are mainly based on the clinicopathological problem the hematopathologist is faced with, for example, the differential diagnosis of reactive lymphadenopathy, the state-of-the-art classification of acute leukemia, including all required ancillary diagnostic techniques, the effect of previous therapy on the bone marrow, or the detection of metastatic tumor in the bone marrow.

In addition to the information on clinical presentation and prognosis that is given in the parts describing individual disease entities, a more clinically oriented chapter provides information on risk stratification and current therapies for the acute leukemias, including tyrosine kinase inhibitors and monoclonal antibodies.

The chapter on expression profiling in acute leukemia received disproportionate space due to excessive technical details and a historical overview with pictures of several generations of cDNA arrays. This is made up for by the critical discussion of the potential and pitfalls of expression profiling in diagnostics and an update of ongoing studies to evaluate the real potential of this new tool for diagnosis, therapy stratification, and prognosis.

A very well-illustrated chapter discusses histiocytic proliferations in children, including the use of recently available antibodies, and the macrophage activation and hemophagocytic syndromes that can be a clinicopathological challenge.

In summary, this is a long-awaited addition to the bookshelf of every pathologist dealing with pediatric or hematological cases. 